\title{
LESSONS LEARNED IN A CLINICAL TRIAL FOR MILITARY SEXUAL TRAUMA-RELATED POSTTRAUMATIC STRESS DISORDER
}

\author{
Alina Surís, PhD; Nicholas Holder, BS; Ryan Holliday, MA; E. Ellen Morris, PhD
}

\section{$\triangle$} large body of literature exists describing the challenges associated with implementing randomized controlled clinical trials (RCTs) [1]. However, when clinical trials are conducted within Department of Veterans Affairs (VA) Medical Center (VAMC) settings, several additional and unique factors contribute to the difficulty of conducting RCTs. The challenges and strategies to address them, described in this editorial, are based on an RCT conducted to determine the effectiveness of an evidence-based therapy to treat Veterans with military sexual trauma (MST)-related posttraumatic stress disorder (PTSD) [2].

\section{Logistical and Environmental Challenges at VAMCs}

The number of Veterans that VAMCs serve has increased substantially since they were built [3]. Due to the design and age of many VAMCs and the increased demand for care, physical space within many VAMCs is at a premium, often necessitating updates and reorganization that require construction. Some examples of difficulties that were experienced during our study included (1) a large construction project to add needed parking spaces (temporarily reducing existing parking); (2) space issues that required frequent VAMC reorganization and changes in office locations for study therapists and personnel; (3) ongoing construction that produced frequent loud noises; and (4) due to space limitations, female Veterans with MST-related PTSD had to share small waiting rooms with male Veterans prior to study visits, often resulting in distress.

Frustration and distress related to these experiences may have affected the way participants completed self-report outcome measures relating to PTSD, anger, depression, and anxiety symptoms. For example, although assessments asked participants to consider a discrete time period encompassing at a minimum the past few days, changes in current affect and anxiety can influence participants' responses [4-5]. Variability in participation could also have been affected by loud construction noise and encounters with male Veterans in small waiting rooms. These events are similar to exposure elements prominent in some therapies for PTSD, and this unintentional form of exposure could interfere with self-report accuracy.

\section{Proposed Strategies to Address Logistical} and Environmental Challenges

We recommend researchers anticipate logistical and environmental challenges by acquiring information about construction schedules; informing participants about construction challenges before starting the study; considering construction-related appointment delays during scheduling; and attempting to create quiet, private waiting areas. To further address space and parking limitations, reducing visits to the medical center can be helpful. For example, we encourage researchers to be flexible with follow-up data collection, such as offering phone assessments.

\section{Challenges with Documentation in the} VAMC Electronic Medical Record

Documentation of the diagnosis and treatment of PTSD in the electronic medical record (EMR) raised several concerns for our study, including (1) confusion about participation affecting VA disability claims; (2) perception that the diagnosis will create barriers to employment, government security clearances, and/or reenlistment; and 
(3) concerns about how participation in the study would affect access to other VA treatment.

\section{Proposed Strategies to Address Challenges with Charting in the VAMC EMR}

Researchers should address Veteran-specific concerns related to documentation during the informed consent (IC) process. Researchers should provide information on what exactly is documented (e.g., attendance and procedures at a study visit) and not documented (e.g., outcome data that could show improvement or worsening of symptoms). Researchers should also be knowledgeable about how information documented in a participant's EMR may or may not affect obtaining a security clearance, disability, and additional treatment. Producing a fact sheet with specific regulation information and directions to resources for further information may help reassure Veterans and help them make better informed decisions regarding participation. Certificates of Confidentiality can also address privacy concerns and should be considered by researchers before implementing psychotherapy RCTs.

\section{Integration of Research and Treatment within VAMCs}

Often when participating in psychotherapy RCTs, individuals attend sessions at a separate institution from where they access their usual medical or mental health care. This is not the case when Veterans participate in research at the VA as the research team cannot only access participant's EMR, they also often become an integral part of the treatment team and/or consult with the mental health team often.

Challenges can arise with reconciling treatment and research participation after Veterans complete the treatment phase and enter the follow-up phase. Veterans were frequently asked to make a decision between completing study-related follow-up sessions or beginning a new course of treatment or medication. In our RCT, participants were more committed to treatment phases of the study in comparison to follow-up visits, with 31 percent of participants unable/unwilling to complete psychotherapy treatment and 44 percent unable/unwilling to complete all follow-up sessions [2].

\section{Proposed Strategies to Facilitate Integration of Research and Treatment within VAMCs}

Our practice of consulting with participant's mental health treatment teams and/or being a part of the treatment team became a strong benefit during the follow-up phase. We were able to inform the providers about upcoming research follow-up appointments and work as a team to complete the study protocol while still attending to participant's requests.

Another helpful practice is to coordinate study intervention and follow-up appointments with existing VAMC appointments. Having flexibility with the follow-up assessment windows or intervention windows allows for increased chances that appointments will not be missed, as it increases the convenience of attending for the participants and minimizes outside conflicts. Another benefit of scheduling in the same medical care system is the potential for participants to be reminded about upcoming research appointments at general health appointments.

\section{Recruitment at the VAMC-A Series of Unexpected Challenges}

Our initial recruitment plan included a heavy emphasis on using resources within the mental health division of our VAMC. We briefed providers at the PTSDfocused Mental Health Trauma Services team about our study and encouraged them to inform qualifying Veterans about the study. We also made announcements at Veterans' psychotherapy groups as well as the local VAMC Women's Clinic and posted institutional review board (IRB)-approved advertisements within the hospital. However, these recruitment efforts did not yield a large number of study referrals. Many of the Veterans accessing mental health care were not interested in psychotherapy, choosing less time-intensive pharmacological treatments instead. Additionally, the stigma associated with MST continued to be an issue in both male and female Veterans [6-8].

To address recruitment issues, we diversified our recruitment strategies by expanding clinician briefings, with research personnel presenting information about the study to general mental health clinics. Additionally, we obtained IRB approval to send information letters to female Veterans residing in our VAMC's catchment area, inviting them to call us to discuss the study. This method also enabled us to recruit participants who do not regularly attend appointments at the VAMC. Finally, we opened recruitment to male survivors of MST. These 
efforts substantially increased access to the pool of eligible participants.

\section{Additional Proposed Strategies to Participant Recruitment at VAMCs}

Recent findings suggest that another potentially effective recruitment method is to use research opt-in/ opt-out approaches for the entire Veteran population within researchers' targeted VAMC health care system [9]. In this design, a local VAMC mails either an opt-in letter (reply if you consent to be contacted for research studies) or opt-out letter (reply if you do not consent to be contacted for research studies) to create a large pool of potential participants. Because of recruitment challenges in our study and to prepare for future research efforts, we secured IRB approval for a PTSD research registry. Veterans attending appointments in Mental Health Trauma Services are offered an opt-in or opt-out opportunity to enroll in the registry to be contacted regarding future PTSD-related research. Our registry has been successful with more than $\mathbf{8 0 0}$ Veterans enrolled to date.

\section{Special Considerations for IC with Veterans}

Research participation requires careful and extensive IC, both before consenting and during the study; however, the IC process can be especially important when working with Veterans. Proper IC requires that research personnel are confident that potential participants understand the research study that they are considering joining. Because Veterans are accustomed to superior-subordinate relationships, they may not have a sense of their ability to decline participation or potential alternatives to participation [10]. Misunderstandings regarding these rights may lead Veterans to not being active and collaborative in the IC process. Because the potential participant could be consenting out of a sense of duty rather than true interest, it may result in decreased participation in the actual study.

\section{Proposed Strategies to Improve the IC Process for Veterans}

A useful method to assure understanding during the IC process is to assess the potential participant's understanding of the study. This can be accomplished in a routine manner by asking the participant to describe the research design and their rights. Additionally, as previously discussed, researchers can also consider providing potential participants with a brief, supplementary fact sheet regarding their rights and what to expect in the study. This fact sheet would ideally help address study-related concerns including the possible effects of participating in the study on future/ current benefits, employment, and VAMC mental health treatment.

\author{
Alina Surís, PhD; ${ }^{1-2 *}$ Nicholas Holder, BS; ${ }^{1-2}$ \\ Ryan Holliday, MA; ;-2 $^{1-2}$. Ellen Morris, PhD $^{1}$ \\ ${ }^{1}$ Department of Veterans Affairs North Texas \\ Health Care System, Dallas, TX; ${ }^{2}$ University of Texas \\ Southwestern Medical Center, Dallas, TX \\ *Email: alina.suris@va.gov
}

\section{REFERENCES}

1. Imel ZE, Laska K, Jakupcak M, Simpson TL. Meta-analysis of dropout in treatments for posttraumatic stress disorder. J Consult Clin Psychol. 2013;81(3):394-404. [PMID:23339535] http://dx.doi.org/10.1037/a0031474

2. Surís A, Link-Malcolm J, Chard K, Ahn C, North C. A randomized clinical trial of cognitive processing therapy for veterans with PTSD related to military sexual trauma. J Trauma Stress. 2013;26(1):28-37. [PMID:23325750] http://dx.doi.org/10.1002/jts.21765

3. Rosenheck RA, Fontana AF. Recent trends In VA treatment of post-traumatic stress disorder and other mental disorders. Health Aff (Millwood). 2007;26(6):172027. [PMID:17978391] http://dx.doi.org/10.1377/hlthaff.26.6.1720

4. Huprich SK, Bornstein RF, Schmitt TA. Self-report methodology is insufficient for improving the assessment and classification of Axis II personality disorders. J Pers Disord. 2011;25(5):557-70. [PMID:22023295] http://dx.doi.org/10.1521/pedi.2011.25.5.557

5. Schwarz N, Clore GL. Mood, misattribution, and judgments of well-being: Informative and directive functions of affective states. J Pers Soc Psychol. 1983;45(3): 513-23.http://dx.doi.org/10.1037/0022-3514.45.3.513

6. Turchik JA, McLean C, Rafie S, Hoyt T, Rosen CS, Kimerling R. Perceived barriers to care and provider gender preferences among veteran men who have experienced military sexual trauma: A qualitative analysis. Psychol Serv. 2013;10(2):213-22. [PMID:22984877] http://dx.doi.org/10.1037/a0029959

7. Morris EE, Smith JC, Farooqui SY, Surís AM. Unseen battles: The recognition, assessment, and treatment issues of men with military sexual trauma (MST). Trauma 
ViolenceAbuse. 2014;15(2):94-101. [PMID:24231941] http://dx.doi.org/10.1177/1524838013511540

8. Turchik JA, Bucossi MM, Kimberling R. Perceived barriers to care and gender preferences among veteran women who experienced military sexual trauma: A Qualitative Analysis. Mil Behav Health. 2014;2(2):180-88. http://dx.doi.org/10.1080/21635781.2014.892410

9. Kaufman D, Bollinger J, Dvoskin R, Scott J. Preferences for opt-in and opt-out enrollment and consent models in biobank research: A national survey of Veterans Administration patients. Genet Med. 2012;14(9):787-94. [PMID:22538255]

http://dx.doi.org/10.1038/gim.2012.45

10. McManus J, Mehta SG, McClinton AR, De Lorenzo RA, Baskin TW. Informed consent and ethical issues in military medical research. Acad Emerg Med. 2005;12(11):
1120-26. [PMID:16264083]

http://dx.doi.org/10.1111/J.1553-2712.2005.tb00839.x

This article and any supplementary material should be cited as follows:

Surís A, Holder N, Holliday R, Morris EE. Lessons learned in a clinical trial for military sexual traumarelated posttraumatic stress disorder. J Rehabil Res Dev. 2016;53(4):vii-x.

http://dx.doi.org/10.1682/JRRD.2016.04.0064

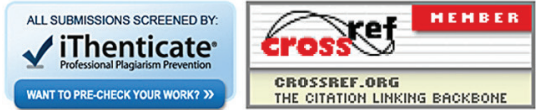

